\title{
Carnets
}

Revue électronique d'études françaises de l'APEF

Deuxième série - 19 | 2020

Petite fabrique d'interprètes

\section{Haddock et le contenu alcoolique de l'aventure}

\section{Cristina Álvares}

\section{(2) OpenEdition}

Journals

Édition électronique

URL : http://journals.openedition.org/carnets/11111

DOI : 10.4000/carnets.11111

ISSN : 1646-7698

Éditeur

APEF

Référence électronique

Cristina Álvares, « Haddock et le contenu alcoolique de l'aventure », Carnets [En ligne], Deuxième série 19 | 2020, mis en ligne le 31 mai 2020, consulté le 23 décembre 2020. URL : http:// journals.openedition.org/carnets/11111; DOI : https://doi.org/10.4000/carnets.11111

Ce document a été généré automatiquement le 23 décembre 2020.

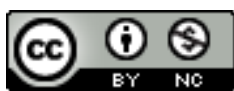

Carnets est mis à disposition selon les termes de la licence Creative Commons - Atribution - Pas d'utilisation commerciale 4.0 International. 


\title{
Haddock et le contenu alcoolique de l'aventure
}

\author{
Cristina Álvares
}

\section{L'eau et le feu ou le (dé)goût de Haddock}

1 Au début des Sept oules de cristal (1948), un petit épisode comique nous montre le Capitaine Haddock qui rate un tour d'illusionnisme consistant à changer de l'eau en vin. Il l'avait vu exécuter par un illusionniste dans un spectacle auquel il assistait tous les soirs depuis quinze jours dans l'espoir de découvrir le truc magique et de le recréer à la maison. Son objectif est de s'assurer que l'eau deviendrait du vin, que l'alcool ne manquerait pas. Changer de l'eau en vin relève du miracle et de la magie. Tout en évoquant le miracle christique des noces de Cana, changer de l'eau en vin c'est changer de l'eau en feu puisque l'alcool (le vin, le whisky, le rhum, le cognac, l'aguardiente) est bel et bien du feu liquide, du feu à boire. La transmutation des éléments et des substances est l'affaire de la magie et en effet la magie, en l'occurrence la magie sympathique $^{1}$, est au cœur de cette histoire.

2 Ce petit épisode comique présente le personnage de Haddock sous le registre du pathos et du thymos pour autant qu'il concerne le goût et le dégoût de Haddock. L'eau le dégoûte, il la crache, alors que l'alcool l'attire irrésistiblement, il ne peut pas s'en passer. Simultanément, l'épisode associe l'irrépressible tendance alcoolique du personnage à ce qui n'a pas de consistance ontologique: le spectacle, le leurre, le mirage (l'illusionnisme). Dans cet album la connexion entre addiction et aliénation prend la forme inquiétante de l'hallucination provoquée par une substance narcotique à base de coca qui se répand quand des mystérieuses boules de cristal éclatent. Au-delà de son association traditionnelle à la magie, la boule de cristal renvoie à la figure de la sphère ainsi qu'à la dialectique du contenant et du contenu, ce pour quoi nous aurons recours à la théorie des sphères de Peter Sloterdijk. 


\section{La figure héroïque : Haddock extension de Tintin}

3 Avec la création du Capitaine en 1941, dans Le crabe aux pinces d'or, Hergé a ajouté un troisième personnage à une figure héroïque déjà composée de Tintin et Milou. On a remarqué que la présence de Haddock auprès de Tintin a progressivement réduit le rôle de Milou, les deux amis du héros partageant un certain nombre d'inclinations, notamment le goût pour le repos et le penchant alcoolique (cf. Verrier, 2013: 91, 99-105). L'attribution de propriétés humaines ou, en l'occurrence, de vices humains à un personnage non-humain comme Milou est, comme l'a bien montré Dipayan Mukherjee, une stratégie anthropocentrique d'appropriation et de domestication de l'altérité d'un animal, typique du making pets (cf. Mukherjee, 2016: 215-217). Or, il semble que cette connexion entre Milou et le Capitaine relève d'une altérité irréductible, d'un (reste) impossible à domestiquer et à contenir. Si l'aventure est domestication du monde (découverte, exploration, connaissance), réduction de son inconnu, de son opacité, de son étrangeté, si l'aventure est domestication de l'être et, par conséquent, une valeur humaniste majeure, alors il faut bien reconnaitre que Haddock apporte quelque chose d'inassimilable à la visée de l'aventure.

4 Nous ne pensons pas que Haddock soit l'alter ego ou le faire-valoir (sidekick) de Tintin. Il n'est pas son masque ou son double et sa fonction n'est pas réductible aux maladresses et aux colères mettant en valeur par contraste les qualités du héros. La figure héroïque composée du protagoniste et du compagnon me semble découler moins du comics américain que de la tradition littéraire des paires comme Roland et Olivier, Pantagruel et Panurge, D. Quichotte et Sancho Panza, D. Juan et Sganarelle, Holmes et Watson. Cette tradition se prolonge dans la BD avec, parmi beaucoup d'autres, Spirou et Fantasio ou Astérix et Obélix, et aussi dans les séries télévisées avec White and Pinkman (Breaking Bad), pour donner un exemple assez récent. Les personnages qui forment ces couples dans une conjonction logico-fonctionnelle incarnent des faisceaux contrastants de propriétés en tension dialectique (cf. Álvares, 2017: 224). À la figure des paires littéraires, nous pouvons ajouter celle des corps connexes que Pierre Sterckx a spécifiquement étudié dans Tintin. Dans son essai Tintin Schizo, Pierre Sterckx considère que les personnages de Haddock, Tournesol, Castafiore, Dupond et Dupont, sont des "corps connexes» de Tintin chacun incarnant une dimension (un visage) du protagoniste (2007: 14). Nous nous inspirons de ces deux figures, l'une puisée dans la tradition littéraire, l'autre dans un ouvrage théorique portant sur Tintin, pour cerner Haddock comme une extension de Tintin, tout en soulignant la tension qu'il y a dans le mot extension.

\section{Pure forme (Tintin)}

Quelle est alors la tension qui cimente le lien de pure amitié qui unit les deux personnages et quel rôle joue-t-elle dans l'univers tintinesque ? Chaque personnage a une attitude différente face à l'aventure : Tintin toujours prêt à partir et à s'exposer à l'étrangeté et à l'hostilité du monde; Haddock préférant la tranquillité et le repos à Moulinsart. L'opposition entre dynamique et inertie ou entre nomadisme et sédentarité ou encore entre monde et maison retentit dans l'opposition entre liberté et dépendance. Dépendance alcoolique pour le Capitaine, alors que Tintin est superbement autonome, libre de tout ce qui attache, sédentarise et fait appartenir, 
entièrement disponible à courir le monde. Possédant un cœur pur, plein de compassion et de générosité, et un caractère solide et sobre, il mène une lutte tenace contre les narcotrafiquants et autres criminels et se tient à l'écart des substances toxiques, alcool ou drogues, dont l'effet aliénant saperait le désir d'aventure. Tintin ne boit que de l'eau minérale.

6 Ce profil clean prend tout son éclat si on tient compte que Tintin est un personnage inédit dans l'histoire de la fiction et dans l'imaginaire héroïque. Son originalité tient au fait qu'il a n'a pas de contenu biographique ou psychologique. Il n'a pas de vie en dehors de l'aventure, car sa vie est entièrement subsumée dans l'aventure. Aussi son contenu est-il extériorisé sans reste dans l'étendue et l'action, ce qui fait de lui un cogito évidé de tout contenu et soutenu par le seul désir d'aventure. Il est intéressant de remarquer un deuxième trait inédit corrélatif du premier : Tintin n'a pas non plus de contenant dans la mesure où aucune sphère domestique et familiale ne l'a enveloppé et, dans les termes de Sloterdijk, insularisé. L'insulation est le terme forgé par le philosophe allemand pour désigner la formation de sphères, espaces ronds dotés d'un intérieur et d'un effet immuno-systémique (cf. Sloterdijk, 2010 : 31). Le prototype en est la sphère maternodomestique, prolongement utérin qui supplée à la néoténie et assure protection et sécurité à l'être par la mise à distance du dehors à partir d'un bord qui délimite le dedans matériellement configuré dans la maison (le foyer). La sphère maternodomestique est le lieu majeur de l'insulation ou « domestication de l'être ». La capacité à créer des sphères qui amortissent l'agression du monde, qui la tiennent à l'extérieur, à distance, a été décisive pour l'évolution de l'espèce et constitue le propre de l'existence humaine (ibid. : 51). Pour Sloterdijk, l'être-dans-le-monde c'est l'êtredans-les-sphères, la sphère étant non seulement la condition de l'invention de l'humain, mais aussi la modalité spécifiquement humaine d'habiter le monde. «(...) la forme de vie plus risquée qui s'est imposée dans l'évolution [est] ce que l'on n'aurait pas pu obtenir sans un surcroît de sécurité en un autre endroit » (Sloterdijk, 2010 : 121-122). Autrement dit, toute exposition (de l'espèce ou de l'individu) à l'ouvert et à l'indéterminé du monde, et c'est bien en cela que consiste l'aventure, présuppose l'insulation comme sa condition de possibilité.

Or, il faut bien le dire, ce qui caractérise et singularise Tintin dans l'imaginaire hérö̈que c'est précisément qu'il est un héros produit sans insulation (cf. Álvares, 2020). Il n'a pas d'origine, pas de passé, pas d'enfance, pas de famille, pas de vie privée. En l'absence de tout drame familial ou récit des origines, on ne saurait dire comment sa bulle première a éclaté, comment s'est opéré sa transition entre dedans et dehors, comment il s'est trouvé exposé au monde. Il l'est toujours déjà. Rien ne le contient, ne l'attache, ne le borne. Sans contenu et sans contenant, Tintin est le comble du sujet moderne, autonome et transparent ${ }^{2}$, un sujet qui est, disons, pure forme, identique à la forme de l'aventure.

\section{Substance toxique (Haddock)}

8 On ne peut dire autant du Capitaine Haddock. Lorsque Tintin le rencontre dans Le crabe aux pinces d'or, nous apprenons qu'il a (ou a eu) une mère et qu'il a un passé. Prisonnier dans son propre navire, alcoolisé et infantilisé, Haddock pleure pour sa maman et s'accuse d'être un misérable. Cet indice d'insulation et de drame familial, centré sur la référence maternelle, apparait dans un cadre pathétique d'humiliation et de culpabilité 
qui ne sera jamais éclairé, d'autant plus que la mémoire familiale de Haddock va quitter la sphère maternodomestique pour la sphère bien plus large, fière et prestigieuse du lignage de François de Hadoque, chevalier au service de Louis XIV, héros d'aventures maritimes. Dans Le Secret de la Licorne (1943), le registre pathétique passe au registre héroïque des aventures de l'ancêtre, en même temps que la mémoire subjective désadhère de la vie privée pour se hausser à la mémoire historique (cf. Álvares, 2017 : 220-221) ${ }^{3}$. Mais ces déplacements ne viendront pas à bout de l'alcoolisme. Si le whisky est associé à la dépression indexée à la mère absente, le rhum imprègne le récit euphoriquement dramatisé des aventures de l'aïeul, au point que le narrateur vient à s'identifier au personnage, Haddock mimant Hadoque, dans une séquence qui alterne narration et récit et qui suggère que le goût des voyages maritimes et des aventures est génétique.

9 La différence entre les deux personnages, l'un produit sans insulation, l'autre avec, se traduit dans l'élément attribué à chacun: l'eau pour Tintin, le feu (l'alcool) pour Haddock. L'eau exprime la sobriété de Tintin (sa cleanness), cogito sans substance, tandis que le feu indique la nervosité du Capitaine : colères, paniques, hyper-sensibilité à la présence de la Castafiore. À l'égard du seul personnage féminin des Aventures de Tintin, on remarquera que le miracle christique des noces de Cana associe le vin au mariage, feu alcoolique et feu sexuel étant solidaires, et que dans Les ijoux de la Castafiore il sera question du mariage de Haddock avec la diva. Elle apparait très fugacement dans Les sept boules de cristal où le Capitaine compare la puissance de sa voix à celle d'un ouragan. L'allusion à un miracle qui associe le vin au mariage est une façon de sexualiser l'alcoolisme du Capitaine Haddock, ou plutôt de pointer la dimension sexuelle du noyau immaitrisable, indisciplinable, irrésistible, excessif de toute pulsion. Mais au-delà de la touche sexuelle qu'elle introduit dans l'addiction, la référence évangélique fait flotter l'ombre du mariage au-dessus de Haddock. Nous disons "l'ombre » parce que l'univers fictionnel de Tintin se caractérise par l'inexistence de liens de sang ou de sexe, la question nuptiale ne se posant jamais, sauf pour Haddock mais de manière très elliptique et oblique dans Les bijoux de la Castafiore (1962) où la perspective du mariage fait paniquer le Capitaine. Heureusement pour lui, l'annonce des fiançailles s'avère finalement n'avoir d'autre ancrage que médiatique, ne servant qu'à des fins d'autopromotion de la femme du spectacle, la diva vivant d'apparences (encore de l'illusionnisme).

10 En somme, si la seule passion de Tintin est l'aventure, Haddock est là pour signifier qu'il y a des passions en dehors de l'aventure.

\section{Contenir la substance toxique dans la forme de l'aventure}

11 La tension à l'œuvre dans la figure héroïque composée de Tintin et d'Haddock n'est pas réductible à l'opposition élémentaire eau-feu/alcool. Celle-ci doit être envisagée dans le cadre de l'aventure tintinesque où la remise en ordre du monde (d'un petit segment du monde) passe souvent par le combat aux narcotiques (Les cigares du pharaon, Le lotus bleu, Le crabe aux pinces d'or, Les sept boules de cristal, Coke en stock). En effet l'action de Tintin, qui défait impostures, illusions, énigmes et mythes, pour rétablir le sens de la réalité et dans la réalité, constitue une désintoxication (réelle ou métaphorique) contre toute forme d'aliénation, d'évasion, de torpeur. L'aventure est tonique et le héros 
manifeste une vitalité, une confiance et un optimisme inébranlables. Or ce que Haddock apporte à l'aventure c'est la toxicité de la substance. Étienne Verrier écrit: "Par exemple, le capitaine Haddock et Milou sont foncièrement du côté du Bien mais leurs errements, bachiques pour le premier, de diverse nature pour le second, les font passer transitoirement dans le camp opposé » (Verrier, 2010 : 71). À notre avis, il s'agit moins de passage transitoire dans le Mal que d'introduire la substance toxique au sein de la figure héroïque, c'est-à-dire du Bien - ce qui en quelque sorte rend le Bien moins bien.

Par conséquent, l'alcoolisme du compagnon pose un problème à Tintin : comment contenir l'excès, le déversement, la toxicité de la substance aliénante dans la forme de l'aventure qui tient à la réalité. Contenir signifie limiter, réduire pour maîtriser. Tintin n'aura de cesse d'essayer de discipliner la consommation d'alcool en la réduisant à un niveau socialement admissible. L'histoire du Crabe est une suite de bêtises, causées par l'alcoolisme du Capitaine, qui mettent les personnages en danger et créent des obstacles et des impasses à l'action. Par exemple, Haddock hallucine que Tintin est une bouteille de Champagne qu'il essaie d'ouvrir en l'étranglant. Cet album s'achève sur un malaise provoqué par l'ingestion accidentelle de l'eau. Les albums suivants nous présentent un Capitaine qui ne part pas en aventure sans s'approvisionner en whisky (l'alcool est ce qui ne saurait manquer) mais qui reste assez sobre quand même. Ces oscillations signalent un difficile exercice d'autodiscipline déstabilisée ici et là par des retombées passagères. Dans L'étoile mystérieuse (1942), la séquence où la soi-disant goutte de whisky que Haddock souhaite ajouter à sa triste eau minérale finit par déborder le verre, signifie que tout projet de contenir la substance toxique est voué à l'échec. Et pourtant Haddock réussit à boire du rhum de Jamaïque vieux de 300 ans sans se soûler ; l'aguardiente sudaméricaine est trop forte pour lui (il y a trop de feu); et lorsqu'il hallucine que le portrait de Tournesol bouge et parle, c'est volontairement qu'il renonce à boire son verre de whisky. Contention et modération?

\section{Des sphères fragiles}

13 L'aventure de la lune fait basculer cet équilibre précaire, parce qu'elle conjoint dépaysement radical et alcool formellement interdit. En Syldavie, pays de la grande entreprise technoscientifique du voyage à la Lune, l'alcool est banni et dans la fusée aussi et encore plus catégoriquement (Hergé, $1953: 3$, 53). Or, Haddock s'approvisionne clandestinement en bouteilles de whisky comme pour n'importe quel voyage (Hergé, 1954 : 4). Dans un premier temps, il n'arrive pas à boire parce que sous l'effet de l'apesanteur le contenu du verre prend la forme d'une boule (ibid. : 5). Nous retrouvons la sphère. En effet, que sont les bouteilles de whisky dont Haddock se pourvoit pour chaque aventure sinon des sphères contenant la substance qui amortit l'hostilité du monde auquel il s'expose? La bouteille et le verre de whisky sont les sphères avec lesquelles Haddock habite le monde ou, en l'occurrence, l'espace sidéral, le dehors radicalement inhabitable. Que la substance devienne forme et le contenu contenant est bien le signe de l'inhabitable de la fusée et de l'espace lors de ce voyage inédit où, pour la première fois, on a marché sur la lune. Dans toutes les autres aventures, sur terre et sur mer, dans le désert ou la jungle, montagnes ou grottes, des bouteilles et des verres tombent, se brisent, éclatent sous des tirs ou des ondes sonores ou tout simplement disparaissent. Mais jamais la perte de whisky n'avait pris la forme de boule. Dans ce casci, la boule donne forme à l'impossibilité de bulle immunisante ou aliénante. C'est la 
(anti-)sphère qui dé-sphérise le monde de Haddock en le soustrayant à la dialectique du dedans et du dehors. Quand la gravité est rétablie et le contenu rentre dans le contenant, Haddock peut enfin se soûler. Il décide alors de quitter la fusée pour revenir au château de Moulinsart (ibid.: 8). Dans l'expérience traumatique du dehors inhabitable la tendance sédentaire émerge sur la tendance alcoolique. Dès lors c'est à contre-cœur que le Capitaine suivra Tintin dans d'autres aventures - il aurait préféré rester tranquille à la maison - et cette inertie du compagnon résiste à la dynamique du protagoniste. Tout se passe effectivement comme si la radicalité de l'expérience lunaire avait mis en évidence l'extrême fragilité des sphères, ces espaces ronds qui rendent la réalité supportable et le monde habitable. D'où une nouvelle valorisation de l'habitation, sphère domestique où Haddock rêve de se détendre à l'abri des turbulences du monde en buvant son whisky. On voit que contrairement à Tintin, Haddock a un contenu (l'alcool) et un contenant (la maison). Il est tiré de cette aliénation toxico-domestique par l'intervention de deux facteurs : l'amitié pour Tintin qui l'entraine malgré lui dans « des galopades autour du monde » (Hergé, 1956:1) et l'intrusion à Moulinsart de personnages intraitables comme Abdallah (Coke $)^{4}$, Lampion (L'affaire, Coke, Les bijoux) et la Castafiore (Les bijoux), qui tous font éclater la sphère domestique et la rendent inhabitable.

\section{Conclusion}

14 Le fait que la fragilité des sphères s'étend au château marque l'entrée de Tintin dans l'ère post-moderne portée par la désphérisation du monde. Après la globalisation terrestre (voyages maritimes, révolution scientifique, colonisation), œuvre de la modernité ayant établi la forme ronde du monde, l'accélération de la circulation des images et des marchandises a remplacé la rondeur du monde par des réseaux en expansion continue. L'image morphologique du monde n'est plus la sphère, mais l'écume, le nuage, le tourbillon et autres phénomènes inconsistants, irréguliers, informes et chatoyants (cf. Sloterdijk, 2010a : 72-82). Bâti au XVII ${ }^{\mathrm{e}}$ siècle, le château de Moulinsart représente la façon moderne d'habiter le monde. Il donne forme architecturale à la continuité de la modernité depuis les temps du Roi-Soleil. Il est le lieu auquel les héros retournent après l'aventure, le retour signalant la circularité du monde. Que les héros puissent se reposer entre deux aventures confirme la stabilité de la rondeur du monde que celle de la sphère domestique reproduit en plus petit. Mais déjà en rentrant du Pays de l'or noir, Tintin et Haddock n'ont pas le temps de se reposer et repartent immédiatement, cette fois-ci en Syldavie pour la grande aventure de la Lune (1953 : 1-2). La circulation s'accélère à la veille d'un voyage inédit lors duquel les personnages percevront la Terre-Maison à très grande distance, jetés qu'ils sont dans l'extériorité radicale de l'espace sidéral (ibid. : 4). Dans les années 1950, la hantise de la destruction du château (1950:62), intensifiée par des intrus suprêmement agaçants qui déstabilisent, voire carnavalisent son ordre interne $(1956: 3-11,13,61 ; 1958: 6,62$; $1962: 8-56$ ), figure, on ne peut mieux, l'extrême fragilité de la bulle domestique dans un monde où tout contenant éclate en écume. 


\section{BIBLIOGRAPHIE}

ÁlVARES, Cristina (2017). « Les nerfs du Capitaine. La fonction du compagnon inséparable du héros dans Les aventures de Tintin de Hergé », Cincinnati Romance Review, 43, pp. 216-225.

ÁLVARES, Cristina (2020). « Hergé dans la théorie des sphères. Problématique de l'insulation et de l'animalité humaine dans Les Aventures de Tintin », Revue Romane, 55, 1, pp. 164-181.

HERGÉ (1941). Le Crabe aux pinces d'or. Bruxelles : Casterman.

HERGÉ (1942). L'Étoile mystérieuse. Bruxelles : Casterman.

HERGÉ (1943). Le Secret de la licorne. Bruxelles : Casterman.

HERGÉ (1948). Les Sept Boules de cristal. Bruxelles : Casterman.

Hergé (1950). Tintin au pays de l'or noir. Paris : Casterman.

HERGÉ (1953). Objectif Lune. Bruxelles : Casterman.

HERGÉ (1954). On a marché sur la Lune. Bruxelles : Casterman.

HERGÉ (1956). L'Affaire Tournesol. Bruxelles : Casterman.

HERGÉ (1958). Coke en stock. Bruxelles : Casterman.

HERGÉ (1962). Les Bijoux de la Castafiore. Bruxelles: Casterman.

MUKHERJEE, Dipayan (2016). « Domesticating the "Other": An Analysis of the Appropriation of NonHumans by Humanistic Discourse in Herge's The Adventures of Tintin ", Rupkatha Journal on Interdisciplinary Studies in Humanities, 8,1, pp. 214-221.

SLOTERDIJK, Peter (2010). Règles pour le parc humain suivi de La domestication de l'être. Paris : Mille et une nuits.

SLOTERDIJK, Peter (2010a). Bulles. Sphères I. Paris : Fayard.

STERCKX, Pierre (2007). Tintin schizo. Paris : Les Impressions Nouvelles.

VERRIER, Etienne (2010). « Les perroquets, Milou, le yéti et les autres : les animaux dans les aventures de Tintin », Ethnozootechnie, 88, pp. 59-71.

\section{NOTES}

1. La magie sympathique consiste à agir sur un être par des pratiques exercées sur un autre être qui est ou a été en relation d'analogie ou de contact avec le premier. C'est le cas des poupées vaudou. Dans Les sept boules de cristal et Le temple du soleil, les scientifiques de l'expédition ethnographique Sanders-Hardmuth, qui avaient découvert la momie de l'Inca Rascar Capac, ont été les victimes de cette pratique magique : ils subissaient les tortures que le prêtre Inca infligeait aux poupées qui représentaient chacun d'eux.

2. Sloterdijk considère que l'idéal du sujet moderne exprime la névrose de liberté qui est la névrose fondamentale de la culture occidentale (2010: 96).

3. Malgré la famille déprivatisée, toujours est-il que pour le compagnon la vie n'est pas entièrement subsumée en aventure. Sa fonction est d'apporter des fragments de ce dont le héros est dépourvu : des bribes de mémoire, un bout de passé, de la famille, des amours toxiques ou 
phobiques, bref, un soupçon de biographie et de psychologie. Haddock, lui, a des liens, des biens, des souvenirs, des vices, des nerfs, des angoisses. Si Tintin est une surface lisse, polie et souple, alors le compagnon est une extension inégale et rugueuse du protagoniste, alourdie du bric-àbrac pathologique de la vie (cf. Álvares, 2017 : 219-220, 224).

4. Dans Coke en stock, après un tour de plus joué par Abdallah, Haddock finit par accepter de partir avec Tintin au Moyen Orient.

\section{RÉSUMÉS}

Nous examinons dans Les Aventures de Tintin la figure hérö̈que composée des personnages de Tintin et de Haddock afin de décrire la tension qui la dynamise. Prenant comme point de départ les éléments présents dans leurs boissons préférées, l'eau et le feu, nous soutenons que le protagoniste et son compagnon incarnent deux visages du héros: la pure forme de Tintin, personnage sans contenant et sans contenu et la substance toxique que Haddock verse dans l'aventure et qu'il s'agit de contenir (au double sens du mot) au nom de sa tonicité. Pour analyser cette tension, nous avons recours à la théorie des sphères de Sloterdijk, en particulier aux notions d'insulation, de sphère et d'écume.

This abstract examines in Les Aventures de Tintin the morphology of the heroic figure composed by two characters, Tintin and Haddock, and aims to describe the tension that fosters it. Taking as a starting point the elements that compose their favourite drinks, water and fire, and believe that the protagonist and his friend represent the two faces of the hero: the pure form of Tintin, a character with no content nor container, and the toxic substance that Haddock brings to the adventure with no intention to hold it back. Our theoretical perspective comes from Sloterdijk's spherology and its main notions of insulation, sphere and foam.

\section{INDEX}

Mots-clés : personnage, aventure, sphère, substance, forme

Keywords : character, adventure, sphere, substance, form

\section{AUTEUR}

\section{CRISTINA ÁLVARES}

Un. do Minho

calvares[at]ilch.uminho.pt 